\title{
Model Predictive Control Based Energy Management Strategy for a Plug-In Hybrid Electric Vehicle
}

\author{
Jieli Zhang ${ }^{1, a}$, Hongwen $\mathrm{He}^{\star^{1, b}}$ and Ximing Wang ${ }^{2, \mathrm{c}}$ \\ ${ }^{1}$ School of mechanical engineering, Beijing Institute of Technology, Beijing 100081, China; \\ ${ }^{2}$ School of transportation science and engineering, Beihang University, Beijing 100191, China. \\ ajieli_zhang1991@163.com, ${ }^{b}$ hwhebit@bit.edu.cn, cxmhn163@163.com
}

Keywords: model predictive control (MPC), plug-in hybrid electric vehicles (PHEVs), energy management strategy.

\begin{abstract}
In this paper, the model predictive control (MPC) method is researched for energy management problem of plug-In hybrid electric vehicle (PHEV). Multi-step Markov prediction method is selected for the prediction. Dynamic programming (DP) is chosen to solve the optimization problem within the prediction horizon. Through the comparison of MPC result with the results of dynamic programming strategy and a rule-based strategy, it is certified that the control effect of MPC strategy is much better than the ruled-based strategy and close to the global optimal control under DP strategy.
\end{abstract}

\section{Introduction}

The model predictive control (MPC) is a novel control method with the idea of using history data or telemetry to predict the future traffic information [1]. Utilizing the limited preview of the velocity profile as the moving optimization horizon, an MPC controller can maintain computational load within a practical range. The reference [2] developed two MPC-based methodologies based on two different cost functions to solve the fuel minimization problem of the power-split hybrid electric vehicles. The reference [3] provided a comprehensive comparative analysis of three velocity prediction strategies, applied within a model predictive control framework. The reference [4] presented a novel algorithm, which uses information from global position system (GPS) and digital maps to schedule the use of the energy buffer along the planned route, for predictive control of parallel hybrid vehicle powertrains.

\section{Plant Model}

In this paper, a single-axis series-parallel plug-In hybrid electric vehicle (PHEV) powertrain was taken as the research object which mainly includes a conventional internal combustion engine (ICE), an integrated starter generator (ISG) motor, a traction motor and a clutch, as shown in Fig. 1.The ICE is directly connected to the ISG motor.

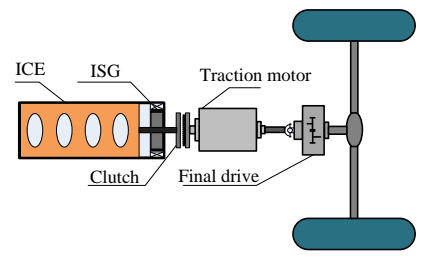

Fig. 1 Powertrain configuration of the PHEV

The fuel consumption rate of the ICE, the traction motor efficiency and the ISG efficiency are extracted from empirical maps, which can be obtained by interpolation functions, shown as follows:

$$
\begin{aligned}
\dot{m}_{e} & =f_{1}\left(T_{e}, n_{e}\right) \\
\eta_{m} & =f_{2}\left(T_{m}, n_{m}\right) \\
\eta_{I S G} & =f_{3}\left(T_{I S G}, n_{I S G}\right)
\end{aligned}
$$

The internal resistance model is the most prevailing model for the charge/discharge process of 
the battery due to its simplicity, described as follows:

$$
\begin{gathered}
P_{b}=V_{o c} \cdot I_{b}-R_{\mathrm{int}} \cdot I_{b}{ }^{2} \\
\mathrm{SOC}=-\left(\frac{I_{b}}{Q_{c}}\right)
\end{gathered}
$$

where $P_{b}$ is the battery power; $V_{o c}$ is the open circuit voltage; $I_{b}$ is the battery current; $R_{\text {int }}$ is the internal resistance; $Q_{c}$ is the maximum battery capacity. Positive $P_{b}$ denotes discharge.

The major external forces acting on a moving vehicle include the rolling resistance of the tires, $F_{r}$; the aerodynamic resistance, $F_{w}$; the climbing resistance, $F_{g}$; and the tractive effort, $F_{t}$. The dynamic equation can be described as follows:

$$
F_{t}=F_{r}+F_{w}+F_{g}+\delta m \cdot \frac{d u}{d t}=m g f_{r} \cdot \cos \alpha+\frac{1}{2} C_{d} \rho A u^{2}+m g \sin \alpha+\delta m \frac{d u}{d t}
$$

where $m$ is the vehicle gross mass; $\delta$ is the mass factor that equivalently converts the rotational inertias of rotating components into translational mass; $\frac{\mathrm{du}}{\mathrm{d} t}$ is the vehicle acceleration; $g$ is the gravity acceleration; $f_{r}$ is the rolling resistance coefficient; $\alpha$ is the road gradient; $C_{d}$ is the aerodynamic drag coefficient; $\rho$ is the air density; $A$ is the vehicle frontal area; $u$ is the vehicle velocity.

\section{Model Predictive Control for Hybrid Electric Vehicle}

The MPC algorithm is a receding horizon optimization strategy based on the predicted information of the system. For PHEV energy management problem, at each simulation step $k$, the steps are taken as follows.

According to historical driving data, a prediction model is built to predict the velocities within a finite horizon, which is defined as the prediction horizon. Therefore, the velocities and accelerations in the prediction horizon are obtained. Then calculate the optimal control sequence within the horizon based on an optimization algorithm. Apply the first element of the optimal control sequence to the vehicle. Update vehicle velocity, and repeat the control procedure.

The PHEV in this paper is a nonlinear, discrete-time system. Vehicle velocity is chosen as the output of the system. SOC is chosen as the state variable and the engine torque $T_{e}$, engine speed $n_{e}$ and motor torque $T_{m}$ are selected as the control variables. The sample interval of MPC is fixed to 1 second. The state transition equation of the PHEV system can be expressed as follows:

$$
x(k+1)=f(x(k), u(k))
$$

where $x(k)$ and $u(k)$ are state vector and control vector, respectively.

For PHEV, the energy management strategies mainly focus on the fuel economy while the electric is much cheaper than the fuel. At step $k$, the cost function is formulated as follows:

$$
\begin{aligned}
J_{k} & =\sum_{t_{k}=k}^{k+p} L\left(x\left(t_{k}\right), u\left(t_{k}\right)\right)+h(x(k+p+1)) \\
& =\sum_{t_{k}=k}^{k+p} \text { fuel }\left(t_{k}\right)+\alpha\left(\operatorname{SOC}(k+p+1)-\operatorname{SOC}_{\mathrm{r}}(k+p+1)\right)^{2}
\end{aligned}
$$

where $L$ is the instantaneous cost function at step $k$ which equals to the instantaneous fuel consumption fuel; $h$ is the terminal cost function of the state variable SOC at the end of the prediction horizon; $\alpha$ is positive penalty coefficient; $\mathrm{SOC}_{\mathrm{r}}$ is the reference value for SOC. At step $k$, the SOC reference value is assumed as following expression:

$$
\operatorname{SOC}_{\mathrm{r}}(k)=\mathrm{SOC}_{0}-\frac{k}{t_{\mathrm{cyc}}}\left(\mathrm{SOC}_{0}-\mathrm{SOC}_{\mathrm{f}}\right)
$$

where $\mathrm{SOC}_{\mathrm{r}}(k)$ is the $\mathrm{SOC}$ reference value at step $k$; $\mathrm{SOC}_{0}$ is the initial maximum SOC value; $\mathrm{SOC}_{\mathrm{f}}$ is the terminal low level of SOC; $t_{c y c}$ is the total travelling time between twice battery charges which is assumed to be known. 
The constraints of system variables can be expressed as follows:

$$
\left\{\begin{array}{l}
n_{e-\text { min }} \leq n_{e}(k) \leq n_{e-\text { max }} \\
T_{e-\text { min }}\left(n_{e}(k)\right) \leq T_{e}(k) \leq T_{e-\max }\left(n_{e}(k)\right) \\
T_{I S G \text {-min }}\left(n_{I S G}(k), \operatorname{SOC}(k)\right) \leq T_{I S G}(k) \leq T_{I S G \text {-max }}\left(n_{I S G}(k), \operatorname{SOC}(k)\right) \\
T_{m-\text { min }}\left(n_{m}(k), \operatorname{SOC}(k)\right) \leq T_{m}(k) \leq T_{m \text {-max }}\left(n_{m}(k), \operatorname{SOC}(k)\right) \\
\operatorname{SOC}_{\text {min }}(k) \leq \operatorname{SOC}(k) \leq \operatorname{SOC}_{\text {max }}(k) \\
n_{m}(k)=n_{e}(k)=n_{I S G}(k) \quad \text { if clutch }=1 \\
n_{e}(k)=n_{I S G}(k) \quad \text { if clutch }=0 \\
T_{e}(k)+T_{I S G}(k)=0 \quad \text { if clutch }=0
\end{array}\right.
$$

A prediction method should be selected for the velocity prediction. We assume that the vehicle acceleration in the future is only related to the current velocity and acceleration and has nothing to do with the historical information, thus the acceleration change process can be seen as a Markov process.

In this paper, the Chinese typical urban drive cycle (CTUDC) is chosen as the sample driving cycle. Take the sample step as 1 second. The velocity and acceleration are discretized into finite values, as shown in Equation 11. The acceleration discrete interval is chosen as $0.05 \mathrm{~m} / \mathrm{s}^{2}$, the velocity discrete interval is chosen as $10 \mathrm{~km} / \mathrm{h}$.

$$
\left\{\begin{array}{l}
a \in\left\{a^{1}, a^{2}, \ldots, a^{r}\right\} \\
v \in\left\{v^{1}, v^{2}, \ldots, v^{l}\right\}
\end{array}\right.
$$

At each moment of the sample driving cycle, known current velocity $v(k)=v^{n}$ and acceleration $a(k)=a^{i}$, record the accelerations of the next $p$ steps, $a(k+1)=a^{j 1}, \ldots, a(k+p)=a^{j p}$. Denoting $m_{n, i, j, x-\text { step }}$ as the number of $x$-step transition from $a^{i}$ to $a^{j}$ at velocity discrete value $v^{n}$. We can get the total number of $x$-step transition from $a^{i}$ at velocity discrete value $v^{n}$, denoting as $m_{n, i, x \text {-step }}$, as shown in Equation 12 At each velocity discrete value $v^{n}$, the $x$-step transition probability from $a^{i}$ to $a^{j}$ is denoted as $P_{n, i, j}$,calculated by Equation 13.

$$
\begin{aligned}
& m_{n, i, x-\text { step }}=\sum_{j=1}^{r} m_{n, i, j, x-\text { step }}, \quad x=1,2, \ldots, p \\
& P_{n, i, j, x-\text { step }}=\frac{m_{n, i, j, x-\text { step }}}{m_{n, i, x-\text { step }}}, \quad x=1,2, \ldots, p
\end{aligned}
$$

Therefore, at each velocity discrete value $v^{n}$, we can get $p$ probability transition matrixes $P_{n, 1-\text { step }}, \ldots, P_{n, p \text {-step }}$. Such $x$-step probability transition matrixes are a total of $l \times p$. Based on current velocity $v(k)$ and acceleration $a(k)$, using $P_{n, 1-s t e p}, \ldots, P_{n, p-s t e p}$ to predict the accelerations $a(k+1), \ldots, a(k+p)$, then obtain the velocities $v(k+1), \ldots, v(k+p)$.

After the predicted velocities within the prediction horizon are obtained, the optimization problem becomes a finite-horizon nonlinear optimization problem with constraints. DP is the best choice to solve this problem. At step $k$, the optimal control sequence is obtained using DP method and only the first control variable is implemented to the vehicle. At step $k+1$, DP is implemented again to obtain the new optimal control sequence.

\section{Simulation Results and Discussion}

In this section, MPC is utilized to solve the energy management strategy problem of the PHEV. The results calculated by MPC are compared with the simulation results of the DP strategy and the PED + HDCD + HDCS strategy from reference [5]. The CTUDC is selected to be used during the 
simulation experiment. The total simulation distance is $180 \mathrm{~km}$, which is attained by repeating 31 consecutive CTUDC cycles. And the vehicle is loaded with $65 \%$ of full load.

At the beginning of the simulation, the battery is assumed as fully charge. Considering the health and efficiency of the battery, the high level and low level are selected to be 0.8 and 0.3 , respectively. When the battery SOC is higher than $80 \%$, the PHEV is not allowable to implement regenerative braking. When implementing the MPC and DP strategy, the $\Delta$ SOC and $\Delta T$ are set to be $0.5 \%$ and $5 \mathrm{Nm}$, respectively [6]. The prediction horizon length for MPC is set to be 10s. The SOC trajectories of the PHEV with three kinds of control strategies are shown in Fig. 2.

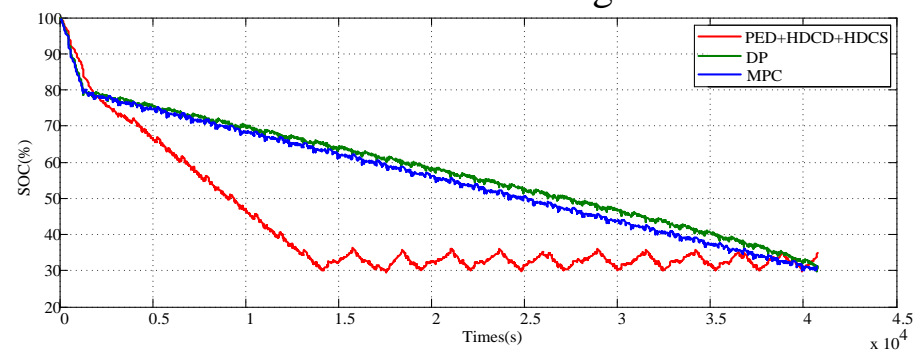

Fig. 2 The SOC trajectories under three kinds of strategies

When the battery SOC is higher than $80 \%$, the tendencies of three SOC trajectories are similar. Since regenerative braking is not available in this area, the SOC decreases rapidly to $80 \%$. When the SOC is between $30 \%$ and $80 \%$, though the SOC trajectory of MPC strategy slightly deviates from DP results, the tendencies of the two strategies are similar. It could be acquired by MPC that the battery SOC approximately linearly decreases to the low level of SOC, just as the DP strategy. Figure 3 shows the relationship between the trip distance and the fuel consumption per $100 \mathrm{~km}$ of the PHEV with three kinds of strategies.

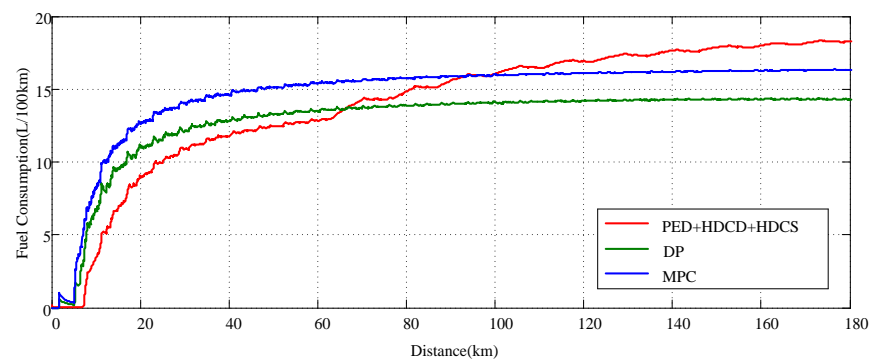

Fig. 3 The relationship curve between fuel economy and trip distance under three kinds of strategies

As shown in Fig. 3, when the trip distance is less than $95 \mathrm{~km}$, the fuel economy of PHEV under PED + HDCD + HDCS strategy is better than the results calculated by MPC and DP strategies. However, the latter is gradually superior to the former as the trip distance increases. Though the MPC strategy is unable to obtain the optimal fuel economy, the fuel economy is improved about $10 \%$ than the PED + HDCD + HDCS strategy.

By the comparative analysis mentioned above, the MPC strategy is obviously better than the PED + HDCD + HDCS strategy and can be implemented to obtain the optimization control close to the global optimal control.

\section{Summary}

The MPC algorithm has been implemented on the energy management problem for PHEV. First, a single-axis series-parallel PHEV was modeled and its systematic model was built for later simulation. Then, we formulated the MPC-based optimization problem. The multi-step Markov method was chosen for the velocity prediction. The global optimization theory is applied to the MPC framework and the DP algorithm was chosen to solve the optimization problem within the finite prediction horizon. The simulation result under MPC strategy was compared with DP strategy and PED + HDCD + HDCS strategy. The MPC result was obviously better than PED + HDCD + HDCS and close to DP result. 


\section{References}

[1] T.S. Kim, C. Manzie and R. Sharma, Model Predictive Control of Velocity and Torque Split in a Parallel Hybrid Vehicle, in: Proceedings of the 2009 IEEE International Conference on Systems, Man, and Cybernetics.

[2] H. Borhan, A. Vahidi and Anthony M. Phillips, MPC-Based Energy Management of a Power-Split Hybrid Electric Vehicle, IEEE Transactions on Control Systems Technology, 2012, 20(3).

[3] C. Sun, X. Hu and S.J. Moura, Velocity Predictors for Predictive Energy Management in Hybrid Electric Vehicles, IEEE Transactions on Control Systems Technology, 2015, 23(3).

[4] L. Johannesson, B. Egardt, A novel algorithm for predictive control of parallel hybrid powertrains based on dynamic programming, in: 5th IFAC Symposium on Advances in Automotive Control, AAC 2007.

[5] X. Wang, H. He and F. Sun, et al, Comparative study on different energy management strategies for plug-in hybrid electric vehicles, Energies, 2013, 6(11): 5656-5675.

[6] X. Wang, H. He, F. Sun. Application Study on the Dynamic Programming Algorithm for Energy Management of Plug- in Hybrid Electric Vehicles, Energies, 2015, 8(4),3225-3244. 